Widal's Typhoid Agglutination Reaction. St. Paul, Minn., Sept. 19, 1897.

To the Editor:-I have read with a great deal of interest an editorial appearing in your issue of September 18, entitled "Evidence which tends to weaken the value of Widal's Typhoid Agglutination Reaction." I had read the discussion upon this subject which took place at the last meeting of the AMERICAN Medical Association, and was very much impressed with the fact that where the most careful work has been done there has seemed to be the least divergence of opinion as to the value of this reaction, and the general nature of the reports made as well as the special report made by the Committee seemed to me to leave but little that could cast any doubt upon the spe. cificity of the reaction. The discussion at the recent meeting of the British Medical Society in Montreal, to which I listened with a great deal of interest and instruction, still more firmly established the value of this test. There would really seem to be but two sources of error in the performance of this test at the hands of competent and careful men. One is the fact that the reaction may be absent upon certain days of the disease; this is really very rarely the case and merely indicates the necessity for making more than the one test where a negative result is obtained. The second lies in the fact that occasionally an im pure culture, supposed to be typhoid, may give misleading reactions.

There can be no doubt that over refinement in technique has led to some errors of observation, and that on the other hand the acceptance of reports from general hospitals where this test has been performed by several different men leads to error of observation for precisely the same reason that we find similar errors constantly occurring under the same condition where other clinical tests are involved. My own experience based upon the use of the very simplest technique as outlined in my papers published in the Medical Record of November 14 and Dec. 5, 1896, has continued to be in all respects favorable to the test which, as I stated at that time, I believe to be worth all the other symptoms of typhoid put together as a means of differential diagnosis. I wrote this letter merely because the editorial question seems to me to unduly magnify certain un. important differences of opinion upon the question of technique and thus obscure the more important fact that there is but little actual difference of opinion as to the immense clinical value of the test. Yours truly,

Chas. Lyman Greene, M.D.

\section{Chelidonium Majus for Inoperable Cancer.}

Chicago, Sept. 20, 1897.

To the Editor:--In a previous number of the Journal I gave an account of the plan of treating inoperable cancer advocated by Dr. Denicenco of Russia. The method consists of injections of two parts of extract of celandin to one of glycerin. The mixture is thrown into the substance of the tumor and also painted on the surface, and some of it taken by the mouth.

Prof. John V. Shoemaker of Philadelphia publishes in the Journat of September 4 an account of five cases in which he tried Denicenco's plan, without getting any effect whatever on the tumors.

On the other hand I tried it in one case, and found that the tumor sloughed out as if destroyed by a caustic, with a great relief of the patient from pain and distress, corresponding closely to the experience of Dr. Denicenco.

Professor Shoemaker's great eminence in pharmacology and therapy insures the carefulness of his tests, yet the total failure to produce any caustic effect in the tumor shows that something was wrong. Perhaps the sample of chelidonium furnished him was inert, or the mixture may have deteriorated after it was made. Dr. Denicenco says his mixture must be constantly renewed, as it rapidly loses strength if kept.
I knew one instance where a well educated physician used for three weeks a fluid extract instead of a solid one, thus making his mixture far too weak. It produced no effect whatever, but when he injected a preparation three times stronger the tumor soon mortified and sloughed out, to the great relief of the patient.

The facts thus far show that a good article, prepared in full strength and not allowed to stand long after the mixture is made, will display its caustic power. It is necessary the herb should be fresh, or at least not very old.

Dr. Denicenco claims that in addition to the local destruction of the primary tumor "the glands become normal." If this is true it is very important, but it seems at first glance at least improbable. I have not had a chance to test the correctness of this statement about the glands, because the latter organs in my case were internal and out of reach.

At present, if there is any value in this plan, it is only for cases which are inoperable, or in which the patient will not consent to operation.

The article of Dr. Denicenco (or Denissenko, as some render the name) first appeared in the Wratch of St. Petersburg.

65 Randolph Street. EDmund Andrews, M.D.

\section{Venesection and Oxygen.}

Chicago, Sept. 20, 1897.

To the Editor :-A propos of the paper by Dr. J. W. Hoff on "Blood-Letting as a Therapeutic Remedy," in the August 28 issue of the Journal, and the letter of Dr. F. Walton Todd in issue of September 18, I am led to relate some of my experiences with blood.letting.

I have perhaps not bled more than a half dozen patients, but have been many times deterred from doing it on account of the popular prejudice against blood-letting.

I have records of two cases which I think especially worthy of report.

The first is that of a man about 30 years old, admitted to Cook County Hospital, Chicago, in August, 1888, during my service as house physician. The patient was suffering from sunstroke. He was unconscious, breathing stertorously, somewhat cyanotic, with pupils dilated and pulse full and bounding. A vein at the elbow flexure was opened and blood allowed to flow until the patient breathed quietly-perhaps twenty to thirty ounces in all. After thirty minutes the patient was conscious and able to talk. Recovery was rapid.

The second case is that of a young man, aged 19. On June 8,1893 , at 9 p. m. I was called and found him suffering great thoracic pain. He had a severe rigor during the afternoon. Temperature $101 \mathrm{~F}$.

June 9-Temperature 101.103, pulse 110 ; delirious. Pneumonitis left lower lobe.

June 10-.Temperature 101, pulse 100. Flighty, but otherwise appears to be doing well.

June 11-Was called in haste at 2:30 a. m. Found the patient gasping for breath; bronchi and throat choked with mucus; respiration 48 , pulse 150 and very weak; cyanosis; axillary temperature $105 \mathrm{~F}$.; patient comatose and could not be roused. Spir. ammon. aromat. and spir. frumenti given hypodermically every fifteen to thirty minutes; appeared to cause improvement in all respects. However, at $7 \mathrm{a} . \mathrm{m}$. he relapsed into a worse state than before; respiration 60 ; lungs, bronchi, trachea and pharynx choked with mucus; pulse 165 per minute, but full ; temperature not taken, but apparently very high.

At this juncture I called Dr. A. J. Ochsner, who resided just across the street, in consultation. We quickly decided that the only possible chance of saving the patient was by venesection, to which the parents consented. Since it appeared to us that the patient could not live even fifteen 